\title{
COMMUTATIVE SEMIGROUP VARIETIES WITH THE AMALGAMATION PROPERTY
}

\author{
G. T. CLARKE
}

(Received 20 May 1980)

Communicated by T. E. Hall

\begin{abstract}
We determine which varieties of commutative semigroups have the weak or strong amalgamation property. These are precisely the varieties of inflations of semilattices of abelian groups.
\end{abstract}

1981 Mathematics subject classification (Amer. Math. Soc.): primary 20 M 07; secondary 08 B 25.

\section{Introduction}

In recent years, several authors have studied amalgamation questions for certain classes of semigroups which form varieties of universal algebras. Imaoka (1976a) has determined which varieties of bands have the weak or strong amalgamation property, and Hall (1978a) has answered the corresponding question for varieties of inverse semigroups. In particular, all varieties of commutative inverse semigroups have the strong amalgamation property. This can be deduced from the proof of Howie (1968), Theorem 3.1; see also Imaoka (1976b).

In another paper (Clarke (1980)), the author has investigated amalgamation questions for varieties of completely regular semigroups, showing in particular that all varieties of orthodox normal bands of abelian groups have the strong amalgamation property. This result follows easily from the proof of Imaoka (1976a), Corollary 2.4.

It seems natural, then, to ask the following question. Which varieties of commutative semigroups have the weak or strong amalgamation property? It was shown by Kimura (1957) that the variety of all commutative semigroups does not have the weak amalgamation property.

CCopyright Australian Mathematical Society 1981 
In this paper, we show that an arbitrary variety of semigroups has the weak or strong amalgamation property if and only if the variety of all inflations of semigroups from that variety has the same property. We also provide a necessary condition, in terms of identities, for a variety of semigroups to have the weak amalgamation property.

From these results we deduce that the varieties of commutative semigroups having the weak or strong amalgamation property are precisely those varieties of semigroups which consist entirely of inflations of commutative inverse semigroups.

\section{Preliminaries}

For background on varieties of semigroups the reader is referred to Evans (1971), on the amalgamation properties to Hall (1979) or Hall (1978b) and on the elementary theory of semigroups to Clifford and Preston (1961).

Result 1. Let $\mathfrak{V}$ be a variety of semigroups, and let $\overline{\mathcal{V}}$ denote the class of all inflations of semigroups from $\mathcal{V}$. Let $\mathcal{C}$ be the variety of null semigroups. Then $\overline{\mathcal{V}}$ is a variety of semigroups; in fact, $\overline{\mathcal{V}}=\mathcal{V} \vee \mathcal{C}$, the join of $\mathcal{V}$ and $\mathcal{C}$ in the lattice of semigroup varieties.

Proof. If $\mathcal{V}$ consists entirely of weakly reductive semigroups, then the result is Proposition 2.6 of Petrich (1974).

Now the varieties of weakly reductive semigroups are precisely the varieties of unions of groups. Suppose that $\mathfrak{V}$ does not consist entirely of unions of groups. Then $\mathfrak{V}$ contains $\mathcal{C}$. Let $A \in \mathcal{V}$. If $B$ is an inflation of $A$, then $B$ is an ideal extension of $A$, by a null semigroup $C$, say, determined by a partial homomorphism. So $B$ is a subdirect product of $A$ and $C$ (see, for example, Lee (1976), Remark 1). Therefore $B \in \mathbb{V}$. Thus $\mathcal{V}$ is closed under the taking of inflations; that is, $\mathfrak{V}=\overline{\mathcal{V}}$.

Resurt 2 (Tamura (1969)). If a semigroup $S$ satisfies an identity $x y=f(x, y)$, where $f(x, y)$ begins in $y$, ends in $x$ and has length at least 3 , then $S$ is an inflation of a semilattice of groups satisfying the same identity.

\section{Inflations of semigroups}

THeOREM 1. Let $\mathfrak{T}$ be a variety of semigroups, and let $\overline{\mathcal{V}}$ denote the variety of 
all inflations of semigroups from $\mathfrak{W}$. Then $\overline{\mathcal{V}}$ has the weak [strong] amalgamation property if and only if $\mathfrak{W}$ has the same property.

Proof. If $\mathcal{V} \neq \overline{\mathcal{V}}$, then $\mathcal{V}$ is a variety of unions of groups. So there exists a positive integer $n$ such that all semigroups in $\mathcal{V}$ satisfy the identity $x=x^{n+1}$.

Let $(S, T ; U)$ be an amalgam in $\overline{\mathcal{V}}$, where $S, T$ and $U$ are inflations of $A, B$ and $C$, respectively with $A, B$ and $C$ all belonging to $\mathcal{V}$. Then (as in Clifford and Preston (1961)) we have $S=\cup_{\alpha \in A} X_{\alpha}$ and $T=\cup_{\beta \in B} Y_{\beta}$, where $\left\{X_{\alpha}: \alpha \in A\right\}$ and $\left\{Y_{\beta}: \beta \in B\right\}$ are families of disjoint sets such that for each $\alpha \in A$ and for each $\beta \in B, X_{\alpha} \cap A=\{\alpha\}$ and $Y_{\beta} \cap B=\{\beta\}$.

Now $A \cap B=C$. For, let $x \in C$. Then $x \in U<S$, and so $\left\{x^{2}, x^{3}, \ldots\right\} \subseteq$ $A$. In particular, $x=x^{n+1} \in A$; and similarly, $x \in B$. Conversely, if $x \in A \cap$ $B$ then $x \in S \cap T=U$, and so $x=x^{n+1} \in C$.

Suppose that the amalgam $(A, B ; C)$ can be strongly embedded in a semigroup $D$ belonging to $\mathcal{T}$. For $\delta \in D$, put

$$
V_{\delta}=\left\{\begin{array}{ll}
X_{\delta} & \text { if } \delta \in A \backslash C, \\
Y_{\delta} & \text { if } \delta \in B \backslash C, \\
X_{\delta} \cup Y_{\delta} & \text { if } \delta \in C, \\
\{\delta\} & \text { if } \delta \notin A \cup B
\end{array}\right\}
$$

Put $W=\cup_{\delta \in D} V_{\delta}$ and extend the product in $D$ to $W$ in the usual way. Then $W$ belongs to $\overline{\mathcal{T}}$, and $(S, T ; U)$ is strongly embedded in $W$.

The other half of the theorem is obvious. The proof for the weak amalgamation case is similar to the above.

Since any semigroup variety of orthodox normal bands of abelian groups has the strong amalgamation property (Clarke (1980)), we now have the following corollary.

COROLlary. Any variety of inflations of orthodox normal bands of abelian groups has the strong amalgamation property.

\section{Commutative semigroups}

Let $U=\{u, v, 0\}$ be a three element null semigroup. Extend the multiplication of $U$ to one of $A=U \cup\{a\}$ by defining $a u=u a=v$ and making all other products equal to 0 . Then $A$ is a semigroup.

LEMMA 1. If a variety $\mathcal{W}$ includes $A$, then $\mathcal{V}$ does not have the weak amalgamation property. 
Proof. Let $B$ be the semigroup obtained by extending the multiplication of $U$ to one of $B=U \cup\{b\}$ in the following way. Put $b v=v b=u$ and all other products equal to 0 . Clearly, $A$ and $B$ are isomorphic.

Now the amalgam $(A, B ; U)$ is not weakly embeddable in any semigroup. For, suppose $W$ is a semigroup containing $A$ and $B$. Then, in $W, u=v b=a u b$ $=a 0=0$.

REMARK 1. This example is a modification, obtained by T. E. Hall, of one due to Kimura (1957).

LeMma 2. Let $A$ be the semigroup of Lemma 1. The variety generated by $A$ is determined by the identities $x y=y x$ and $x^{2}=r s t$.

Proof. It is clear that $A$ satisfies both these identities. Now any nontrivial identity has exactly one of the following forms:

(a) $w=w^{\prime}$, where the words $w$ and $w^{\prime}$ have length at least 3;

(b) $x^{2}=w$, where $w$ has length at least 3;

(c) $x^{2}=y^{2}$;

(d) $x y=w$, where $w$ has length at least 2;

(e) $x=w$, where $w$ is any word.

Any identity from (a), (b) or (c) is implied by the identity $x^{2}=r s t$. Any identity from (d) or (e), other than $x y=y x$, can be shown to imply an identity $x y=w$ with $w$ having length at least 3 . But $A$ satisfies no such identity, since $a u=v$ while $A^{3}=\{0\}$. So $A$ satisfies no identity from (d) or (e) other than $x y=y x$.

THEOREM 2. Let $\mathcal{T}$ be a variety of semigroups, and let $I(\mathcal{V})$ be the set of identities satisfied by all semigroups in $\mathcal{T}$. Then $\mathcal{T}$ has the weak amalgamation property only if $I(\mathcal{V})$ includes an identity of the form $x y=w$, where $w$ is a word of length at least 3.

Proof. From the proof of Lemma 2, it is clear that $A \notin \mathfrak{V}$ if and only if $I(\mathfrak{V})$ includes an identity $x y=w$, where $w$ has length at least 3 . The result then follows from Lemma 1 .

LemMa 3. Let $S$ be a commutative semigroup, and suppose that $S$ satisfies an identity $x y=w$ where $w$ has length at least 3. Then $S$ satisfies an identity of the form $x y=y^{m} x^{n}$, where at least one of $m$ and $n$ is greater than 1 .

Proof. If any variable other than $x$ and $y$ occurs in $w$, then replacing every 
occurrence of every such variable with either $x$ or $y$ gives an identity $x y=$ $f(x, y)$. Suppose that only one of $x$ and $y$ occurs in $f(x, y)$. Then we must have $x y=x^{n}=x z$, whence $S$ is a null semigroup (and therefore satisfies every identity of the form $x y=y^{m} x^{n}, m, n \geqslant 1$ ). Otherwise, we have $x y=y^{m} x^{n}$ where at least one of $m$ and $n$ is greater than 1 .

THeOREM 3. Let $\mathcal{T}$ be a variety of commutative semigroups, and let $I(\mathcal{V})$ be the set of identities satisfied by all semigroups in $\mathcal{W}$. Let $A$ be the semigroup of Lemma 1. The following are equivalent:

(i) $\mathcal{V}$ has the weak amalgamation property;

(ii) $\mathfrak{V}$ has the strong amalgamation property;

(iii) $A \notin \mathfrak{V}$;

(iv) $\mathcal{V}$ consists of inflations of commutative inverse semigroups;

(v) $I(\mathcal{V})$ includes an identity of the form $x y=w$, where $w$ is a word of length at least 3.

Proof. By the corollary to Theorem 1, (iv) implies (ii). Trivially, (ii) implies (i). By Lemma 1, (i) implies (iii); and from the proof of Lemma 2 it follows that (iii) implies (v). The proof will be complete when we show that (v) implies (iv). Let $S \in \mathcal{V}$, and suppose that $S$ satisfies the identity $x y=w$. By Lemma $3, S$ satisfies an identity $x y=y^{m} x^{n}$, where $y^{m} x^{n}$ has length at least 3. By Result $2, S$ is an inflation of a semilattice of groups. So $S$ is a commutative inflation of an inverse semigroup, as required.

\section{Acknowledgements}

I thank my supervisor, Dr. T. E. Hall, for much valuable help and advice. I also thank Dr. P. R. Jones for a number of useful suggestions.

The support of a Monash Graduate Scholarship is gratefully acknowledged.

\section{References}

G. T. Clarke (1980), 'On completely regular semigroup varieties and the amalgamation property", Semigroups, Monash University, Australia, edited by T. E. Hall, P. R. Jones and G. B. Preston, pp. 159-165 (Academic Press, New York).

A. H. Clifford and G. B. Preston (1961), Algebraic theory of semigroups, Volume 1 (Math. Surveys 7, Amer. Math. Soc., Providence, R.I.).

T. Evans (1971), 'The lattice of semigroup varieties', Semigroup Forum 2, 1-43. 
T. E. Hall (1978a), 'Inverse semigroup varieties with the amalgamation property', Semigroup Forum 16, 37-51.

T. E. Hall (1978b), 'Representation extension and amalgamation for semigroups', Quart. J. Math. Oxford Ser. 29, 309-334.

T. E. Hall (1979), 'Inverse and regular semigroups and amalgamation', Algebra Paper 39, Monash University, Melbourne.

J. M. Howie (1968), 'Commutative semigroup amalgams', J. Austral. Math. Soc. 8, 609-630.

T. Imaoka (1976a), 'Free products with amalgamation of bands', Mem. Fac. Lit. Sci. Shimane Univ. Natur. Sci. 10, 7-17.

T. Imaoka (1976b), 'Free products with amalgamation of commutative inverse semigroups', $J$. Austral. Math. Soc. Ser. A 22, 246-251.

N. Kimura (1957), On semigroups (Ph.D. Thesis, The Tulane University of Louisiana).

L. Sin-Min (1976), 'Lattice of equational subclasses of distributive semigroups', Nanta Math. 9, 65-69.

M. Petrich (1974), 'All subvarieties of a certain variety of semigroups', Semigroup Forum 7, 104-152.

T. Tamura (1969), 'Semigroups satisfying identity $x y=f(x, y)$ ', Pacific J. Math. 31, 513-521.

\section{Monash University}

Clayton, Victoria 3168

Australia 